\title{
Dietary pattern and nutrient intakes in association with non-communicable disease risk factors among Filipino adults: a cross-sectional study
}

\author{
Imelda Angeles-Agdeppa ${ }^{1^{* \dagger}} \mathbb{D}$, Ye Sun ${ }^{2^{*}+}$ and Keith V. Tanda ${ }^{1}$
}

\begin{abstract}
Background: This study evaluated the relationship between dietary quality and food patterns of Filipino adults and the rising prevalence of selected cardiometabolic non-communicable disease (NCD) risk factors.

Methods: This is a cross-sectional study that examined the association of dietary pattern and NCDs using data collected in the 2013 National Nutrition Survey. A total of 19,914 adults aged 20 years and above were included in the analyses. The Alternative Healthy Eating Index (AHEI-2010) was used to characterize the dietary quality, and principal component analysis (PCA) was used to identify dietary patterns specific to the study population. Logistic regression models were applied to assess the association between the dietary pattern scores and selected cardiometabolic NCD indices including diabetes, hypertension, dyslipidemia and overweight and obesity with adjustment for potential confounders.

Results: The mean AHEl-2010 score was 19.7 for women and 18.9 for men out of a total possible score of 100 . Three major dietary patterns were identified through PCA: 1) meat and sweetened beverages (MSB); 2) rice and fish (RF) and 3) fruit, vegetables and snack (FVS). After adjustment for potential confounding factors, the AHEl pattern was associated with higher odds of overweight/obesity [extreme-tertile odds ratio (OR) 1.10, 95\% confidence interval (CI) 1.02-1.21]. Subjects in the highest tertile of the MSB pattern had greater odds for overweight/obesity, diabetes, high total cholesterol, low HDL-cholesterol, high LDL-cholesterol, and high triglycerides (OR ranging 1.20 to 1.70, all $p$-value < 0.001). The RF pattern was associated with higher probability of overweight/obesity (OR 1.20, $95 \% \mathrm{Cl} 1.08-1.32)$ high LDL-cholesterol (OR 1.20, 95\% Cl 1.07-1.37), and less likelihood of diabetes (OR 0.87, 95\% Cl 0.77-0.98). The FVS pattern was associated with lower probability of overweight/obesity, diabetes, high triglycerides, and hypertension (OR ranging 0.85 to 0.90 , all $p$-value $<0.05$ ).

(Continued on next page)
\end{abstract}

\footnotetext{
*Correspondence: iangelesagdeppa@yahoo.com.ph; Ye.Sun@rdsg.nestle.com

${ }^{\dagger}$ Imelda Angeles-Agdeppa and Ye Sun contributed equally to this work.

'Department of Science and Technology, Food and Nutrition Research Institute, Bicutan, Taguig, Philippines

${ }^{2}$ Nestlé Research Singapore Hub, Singapore, Singapore
}

(c) The Author(s). 2020 Open Access This article is licensed under a Creative Commons Attribution 4.0 International License, which permits use, sharing, adaptation, distribution and reproduction in any medium or format, as long as you give appropriate credit to the original author(s) and the source, provide a link to the Creative Commons licence, and indicate if changes were made. The images or other third party material in this article are included in the article's Creative Commons licence, unless indicated otherwise in a credit line to the material. If material is not included in the article's Creative Commons licence and your intended use is not permitted by statutory regulation or exceeds the permitted use, you will need to obtain permission directly from the copyright holder. To view a copy of this licence, visit http://creativecommons.org/licenses/by/4.0/ The Creative Commons Public Domain Dedication waiver (http://creativecommons.org/publicdomain/zero/1.0/) applies to the data made available in this article, unless otherwise stated in a credit line to the data. 


\begin{abstract}
(Continued from previous page)
Conclusions: Diet quality of Filipino adults is extremely poor. MSB and RF patterns were associated with a higher risk of cardiometabolic NCD indices, while FVS pattern was associated to lower risks. Identifying healthy and detrimental dietary patterns in the local diet could be informative for future local-based dietary recommendation and area-specific intervention programs.
\end{abstract}

Keywords: Dietary pattern, AHEI, NCD, Adult

\section{Background}

Cardiometabolic syndrome (CMS) is a combination of metabolic dysfunctions mainly characterized by insulin resistance, impaired glucose tolerance, dyslipidemia, hypertension, and central adiposity. People with CMS are two times more likely to die from coronary heart disease and three times more likely to have a heart attack or stroke than those who do not have the syndrome. It is now known that central adiposity is a major contributor to increased cardiometabolic risk [1]. There are many challenges to bringing CMS risk factors under control. However, cardiometabolic programs and therapeutic strategies exist that combine diet and exercise prescriptions and focus on behavioral change to maximize success in reducing cardiometabolic risk factors. These programs have specific recommendations for calorie intake, nutrition, and ongoing cognitive and psychological assessments of habits and unhealthy behaviors [2].

In the Philippines, NCD have overtaken communicable diseases as the top cause of mortality wherein it is estimated to account for $67 \%$ of all deaths in 2016 [3]. The five major NCD in the Philippines in proportion to mortality are cardiovascular diseases (35\%), cancers $(10 \%)$, chronic respiratory diseases (6\%), diabetes (4\%), and other NCD (12\%) [4]. Specifically, diseases of the heart and of the vascular system are the leading cause of mortality in the Philippines [5]. The National Nutrition Survey (NNS) conducted by the Food and Nutrition Research Institute (FNRI) in 2013 showed a large number of Filipinos at risk of selected cardiometabolic NCD factors. Risk factors assessed in the NNS include hypertension, obesity, high cholesterol, and diabetes [6]. In 2014, there were 16 for every 1000 Filipino patients admitted due to a medical condition wherein hypertension was possibly the most common etiology factor [7]. Moreover, in the past decade it has been observed that there is a steady increase in the prevalence of high fasting blood glucose (FBG) from 3.4\% in 2003 to $5.6 \%$ in 2013, and the prevalence is even higher among Filipino adults residing in urban areas (6.4\%).

Food, diet and nutritional status are important determinants of NCD. Poor dietary quality, in particular high salt intake, high saturated and trans-fatty acid intake, and low fruit and vegetable consumption coupled with sedentary lifestyle and stressful environment are some risk factors of CMS development [8]. The role of diet in the etiology of most NCDs is extremely important and considered a modifiable risk factor for NCDs [9]. The Philippines is at a high risk for a rise in NCDs as measured by selected CMS especially among adults since the pattern of consumption among this population group is associated with the consumption of processed food laden with sugar, salt and fat, drinking alcohol, snacking between meals, eating while distracted and sedentary lifestyle [10]. In addition, it has been recognized that dietary patterns rather than single nutrients are stronger predictors of NCD risks, and should be the focus for NCD prevention.

Limited data exist in the Philippines with regards to the local dietary patterns and their associations with NCD. Thus, this study evaluated the relationship between dietary quality and food patterns of Filipino adults and the rising prevalence of selected cardiometabolic NCD risk factors. Through the use of the Alternative Healthy Eating Index (AHEI-2010), which is based on foods and nutrients predictive of chronic disease risk, we could assess the quality of typical Filipino diet. A datadriven approach was also employed to understand major dietary patterns in the population. Using data collected in NNS 2013, dietary patterns derived from both approaches were studied in association with major NCD biomarkers, with the aim to identify potential protective or detrimental dietary patterns using local data that could guide future dietary intervention strategies appropriate and applicable in the Philippines.

\section{Materials and methods \\ Study design and populations}

This study used the data from the 2013 NNS. This is a cross-sectional, population-based survey that characterizes the health and nutritional status, foods consumption and dietary patterns of the Filipino population. The survey used a multi-staged stratified sampling design to represent all 80 provinces of the country covering both urban and rural areas. The first stage of sampling was the selection of Primary Sampling Unit (PSU). A PSU is a barangay or contiguous barangay with at least 500 households. It then follows the selection of Enumeration Areas (EA), a contiguous area in a barangay with $150-200$ households. The final sampling unit is the household. The survey protocol 
was approved by the Ethics Committee of FNRI, and all study participants provided written informed consent.

\section{Data collection}

\section{Demographic and socio-economic data}

Demographic and socio-economic information were collected from the 2013 NNS survey participants, including age, gender, area of residence, marital status, and education. Wealth status of participants was defined by proxy indicators including household possession of vehicles, appliances, materials used for housing construction and sanitation facilities. Scores obtained from principal component analysis were used to define wealth quintiles as poorest, poor, middle, rich and richest.

\section{Dietary data}

The 2 non-consecutive 24-h $(24 \mathrm{~h})$ dietary recall was conducted by registered nutritionist-dietitians through face-to-face interviews in households using structured questionnaires. The interviewer recorded all foods and beverages consumed on the previous day from the moment when they woke up until they went to sleep in the evening. The amount of foods and beverages consumed was estimated using household measures (cups, tablespoons and pieces) or through weighing of food samples. The weights of foods were converted to as purchased values using a portion-to-weight list for common foods compiled by FNRI. If the food was a dish, the interviewee was asked to describe the ingredients of the recipe or name the dish or recipe. The nutrient content of these composite foods were determined by breaking down the different ingredients in the recipe and each was calculated based on INFOODS Guidelines [11].

\section{Derivation of dietary patterns}

We adapted the AHEI-2010 with a priori defined scoring rules to assess the dietary quality of Filipino adults. The scoring criteria for AHEI-2010 were described in detail elsewhere [12]. Briefly, dietary quality was assessed by the intake per day of vegetables, fruit, whole grains, sugar sweetened beverages, nuts and legumes, red/processed meat, fish, alcohol, percentage of energy for polyunsaturated fatty acids (PUFA), and sodium. The intake of each dietary component was scored from 0 (poor diet) to 10 (optimal diet). In the original AHEI-2010 there is an inclusion of trans-fat in the scoring, but this was excluded in our study due to unavailability of trans-fat information in the Philippines Food Composition Table. Therefore, the AHEI-2010 score in our study was the sum of the scores from 10 foods and nutrients components and the total score ranged from 0 to 100 (Table 1).

Principal component analysis (PCA) was used to extract dietary patterns of Filipino adults. Thirty-five major non-overlapping food groups were included in the PCA after variable standardization. The resulting components (dietary patterns) represent combinations of foods consumed by the participants. The number of components retained was based on eigenvalues $(>1)$, the scree plot, and factor interpretability. Varimax rotation was applied in order to obtain the simplest factor structure with improved interpretability. The coefficients defining the linear combinations after the rotation are called factor

Table 1 AHEl-2010 scoring criteria and mean component and total scores among adult men and women. ${ }^{a}$

\begin{tabular}{|c|c|c|c|c|}
\hline Component & Criteria for minimum score $(0)$ & Criteria for maximum score(10) & AHEI-2010 Women & AHEI-2010 Men \\
\hline Vegetables, servings/d & 0 & $\geq 5$ & $0.66 \pm 1.0$ & $0.75 \pm 1.2$ \\
\hline Fruit, servings/d & 0 & $\geq 4$ & $0.56 \pm 1.4$ & $0.50 \pm 1.4$ \\
\hline Whole grains, $g / d$ & & & $0.20 \pm 1.1$ & $0.17 \pm 1.1$ \\
\hline Women & 0 & 75 & & \\
\hline Men & 0 & 90 & & \\
\hline Sugar-sweetened beverages, servings/d & $\geq 1$ & 0 & $2.9 \pm 4.1$ & $2.4 \pm 3.9$ \\
\hline Nuts and legumes, servings/d & 0 & $\geq 1$ & $1.9 \pm 3.6$ & $2.0 \pm 3.8$ \\
\hline Red/processed meat, servings/d & $\geq 1$ & 0 & $1.7 \pm 2.9$ & $1.3 \pm 2.6$ \\
\hline Fish, serving/d & 0 & $\geq 0.2857$ & $6.0 \pm 4.5$ & $6.3 \pm 4.5$ \\
\hline Alcohol, drinks/d & & & $0.04 \pm 0.6$ & $0.11 \pm 0.9$ \\
\hline Women & $\geq 2.5$ & $0.5-1.5$ & & \\
\hline Men & $\geq 3.5$ & $0.5-2.0$ & & \\
\hline PUFA, \% of energy & $\leq 2$ & $\geq 10$ & $0.72 \pm 0.8$ & $0.46 \pm 0.6$ \\
\hline Sodium, mg/d & Highest decile & lowest decile & $5.1 \pm 3.1$ & $4.9 \pm 3.2$ \\
\hline Total & 0 & 100 & $19.7 \pm 7.9$ & $18.9 \pm 8.1$ \\
\hline
\end{tabular}

adapted AHEI-2010; Abbreviations: AHEl: alternative healthy eating index; PUFA: polyunsaturated fatty acids 
loadings and represent the correlations of each food group variable with the dietary component. A factor score was produced for each individual participant for each of the dietary components identified. Prior to PCA, a KaiserMeyer-Olkin test of sampling adequacy (0.5015) and a Bartlett test of sphericity $(p<0.001)$ was performed to assess whether the factor model as a whole was significant. Table 2 are the three components or dietary patterns which were obtained: 1) meat and sweetened beverages pattern (MSB); 2) rice and fish pattern (RF) and 3) fruit, vegetables and snack pattern (FVS).

\section{Anthropometric data and non-communicable diseases biomarkers}

Weight and height of respondents were measured using an electronic calibrated portable stadiometer (SECA) (SECA 217, Hamburg, Germany) and digital double window weighing scale (SECA 874, Hamburg, Germany). Both weight and height measurements were collected twice but a third measurement was taken when two measurements were greater than $0.5 \mathrm{~kg}$ or $\mathrm{cm}$. The mean of the 2 measurements were recorded correspondingly. Body mass index (BMI) was computed as weight (in $\mathrm{kg}$ ) divided by the square of height (in meter). Chronic energy deficiency (CED), overweight (OW) and obesity $(\mathrm{OB})$ were determined using World Health Organization (WHO) definition: BMI < 18.5 for CED; Normal: 18.524.99; OW: $25.0-29.99$ and OB: $>=30 \mathrm{~kg} / \mathrm{m}^{2}$ [13].

Systolic and diastolic blood pressure (BP) measurements were collected by trained nurses prior to blood extraction using a non-mercurial sphygmomanometer (A\&D Um-101TM) and stethoscope in compliance with the Department of Health (DOH) Administrative Order
No. 2008-0021. For every measurement, the mean of two readings taken at least two minutes apart was recorded. Blood samples were collected by trained registered medical technologists (RMT) from the study participants after 10-12 h overnight fasting. Blood samples were first collected using vacutainer tubes with Lithium Heparin for fasting blood glucose and plain tubes for lipids profile, after which they were stored on ice and later centrifuged to separate plasma, alter packed, labelled and frozen at $-20^{\circ} \mathrm{C}$ until ready for analysis in the laboratory. Fasting blood glucose and blood lipids profile (total cholesterol, LDL-cholesterol, HDL-cholesterol and triglycerides) were analyzed using enzymatic colorimetric method with Roche COBAS Integra and Hitachi 912.

Clinical cut-offs were used for each of the biomarkers in the study. Hypertension was defined as systolic $\mathrm{BP}>=$ $140 \mathrm{mmHg}$ and/or diastolic BP $>=90 \mathrm{mmHg}$ according to the 8th Joint National Committee for the Detection, Diagnosis, Treatment and Follow-up of Hypertension [14] Fasting blood glucose was classified based on [15]: $<110 \mathrm{mg} / \mathrm{dL}$ as normal, $110-125 \mathrm{mg} / \mathrm{dL}$ as impaired fasting glucose (IFG), and $>=126 \mathrm{mg} / \mathrm{dL}$ as diabetes. Lipid profile which includes total cholesterol $(<200 \mathrm{mg} /$ $\mathrm{dL}$ as desirable, $200-239 \mathrm{mg} / \mathrm{dL}$ as borderline high, and $>=240 \mathrm{mg} / \mathrm{dL}$ as high), LDL-cholesterol $(<130 \mathrm{mg} /$ $\mathrm{dL}$ as desirable, $130-159 \mathrm{mg} / \mathrm{dL}$ as borderline high, and > $160 \mathrm{mg} / \mathrm{dL}$ as high), HDL-cholesterol $(<40 \mathrm{mg} / \mathrm{dL}$ as low, $40-59 \mathrm{mg} / \mathrm{dL}$ as borderline, and $>=60 \mathrm{mg} / \mathrm{dL}$ as desirable), and triglycerides $(<150 \mathrm{mg} / \mathrm{dL}$ as desirable, $150-199 \mathrm{mg} / \mathrm{dL}$ as borderline, and $>=200$ as high/very high) was assessed using the criteria from Adult Treatment Panel (ATP) III Classification [16].

Table 2 Principal loadings of three major dietary patterns identified among Filipino adults. ${ }^{a}$

\begin{tabular}{lll}
\hline Food Groups & $\begin{array}{l}\text { Meat and Sweetened } \\
\text { Beverages Pattern }\end{array}$ & $\begin{array}{l}\text { Rice and Fish Pattern } \\
\text { Fruit, Vegetables, } \\
\text { and Snack Pattern }\end{array}$ \\
\hline Meat (mainly pork and poultry) & 0.478 & \\
Sweetened beverages & 0.4721 & 0.5688 \\
Rice, noodles \& pasta (mainly rice) & 0.4626 \\
Oils (mainly coconut oil) & 0.3935 \\
Fish \& shellfish & & 0.2746 \\
Eggs \& egg dishes & -0.4187 \\
Other grains (mainly corn grits) & & 0.3761 \\
Fruit & & 0.3412 \\
Nut/pea/bean-based mixed dishes (mainly fried green pea) & & 0.3188 \\
Sugar, syrups, preserves, jams, jellies (mainly sugar) & & 0.3037 \\
Fat (mainly coconut cream) & & 0.2824 \\
Savory snacks (e.g. corn chips, potato chips, fish cracker) & & 0.2775 \\
Vegetables & & 0.2682 \\
Non-alcoholic beverages (mainly coconut water, pure coffee, tea) & & \\
\hline
\end{tabular}

${ }^{a}$ Extraction method: Principal component analysis (PCA) with varimax rotation. Only factor loadings $>0.25$ or $<-0.25$ are shown in the table 


\section{Statistical analyses}

The three PCA-derived dietary pattern factor scores as well as the AHEI-2010 scores were categorized as tertiles according to their distribution in the studied population. Descriptive statistics including means, standard errors (SE) and percentages were used to summarize clinical, social demographics and lifestyle of the participants by tertiles of the dietary pattern scores. Logistic regression analyses were used to test for associations between tertiles of the four dietary pattern scores (independent variables) and the selected CMS (dependent variables). The multivariable model (model 2) was additionally adjusted for total energy intake, age, sex, smoking status, drinking status, urbanity, and wealth status. Trend test across the three tertiles was assessed by modeling the median of each tertile as a continuous variable. Missing data in each variables were excluded in the analysis. All data were analyzed using STATA (version 13; Stata Corp., College Station, TX, USA). The level of significance was set at $P<0.05$.

\section{Results}

For this study, a total of 19,914 adults aged 20 years and above were included in the analyses (men: $n=10,001$ and women: $n=9913$ ), with a mean of age of $45.7 \mathrm{yrs}$. old.

Mean AHEI-2010 score in the studied Philippines adults population was 19.7 for women and 18.9 for men out of a total possible score of 100 (Table 1). This suggested an overall poor quality of diet in the general population. A mean score of 28.2 even in the highest tertile of AHEI-2010 (Table 3) could barely be considered a healthy eating group of subjects. Such lack of variation in the data limited the potential of this hypothesis-based healthy dietary pattern score to differentiate various subgroups of the population. Correspondingly, most of the demographic characteristics of the study participants did not differ significantly across the three tertiles of AHEI-2010 (Table 3). On the other hand, greater differences were observed across the tertile distribution of the three PCA-derived dietary patterns (Table 3). Respondents consuming a MSB pattern (highest tertile) are more likely to be younger, urban residents, from the rich and richest wealth quintiles, nonsmoker, and currently drinking alcohol. The highest tertile of RF pattern are more likely to be younger, males, urban residents, from the rich and richest wealth quintiles, currently smoking and drinking alcohol. Subjects in the highest tertile of the FVS patterns are more likely to be from the richest wealth quintile and less likely to be currently smoking or drinking.

The prevalence of abnormalities in selected cardiometabolic NCD risk factors did not differ significantly across the tertiles of AHEI-2010 score for most measures. In comparison, the highest tertile of MSB pattern was associated with lower prevalence of chronic energy deficiency, hypertension and low HDL-cholesterol, and higher prevalence of overweight, obesity, diabetes, high cholesterol, high LDL-cholesterol, and high triglycerides. The RF pattern was associated with lower prevalence of chronic energy deficiency, hypertension and high LDLcholesterol, and higher prevalence of overweight, obesity, and high triglycerides. The FVS pattern was associated with lower prevalence of diabetes (Table 3).

The intake of energy, total fat and sodium in lowest tertile of AHEI pattern were higher than the intake in the highest tertile, while magnesium, potassium and vitamin $C$ intakes were higher in the highest tertile than the intake in lowest tertile (Table 4). The highest tertile of MSB pattern was associated with higher intakes of energy, total fat, saturated fat (SFA), monounsaturated fat (MUFA), polyunsaturated fat (PUFA), protein, sugar, iron and sodium, and a lower average score of AHEI2010. The intakes of energy, iron, calcium, magnesium, phosphorus, potassium, selenium, and niacin were higher in the highest tertile of the RF pattern than the lowest tertile. For the FVS pattern, the intakes of energy, calcium, fiber, folate, magnesium and potassium were higher than the intakes in the lowest tertile (Table 4).

Logistic regression analyses results of selected cardiometabolic NCD risk factors across tertiles of the 4 dietary patterns are provided in Table 5. After adjustment for various potential confounding factors, the AHEI pattern was associated with higher odds of overweight/ obesity [odds ratio for extreme tertile comparison: 1.1, 95\% CI: 1.02, 1.21]. The MSB pattern was associated with higher odds of overweight/obesity [1.3, 95\% CI: $1.21,1.47]$, diabetes [1.20, 95\% CI: 1.10, 1.36], high total cholesterol $[1.4,95 \%$ CI: 1.29, 1.62], low HDLcholesterol [1.7, 95\% CI: 1.41, 2.10], high LDLcholesterol [1.30, 95\% CI: 1.15, 1.43], and high/very high triglycerides [1.30, 95\% CI: 1.16, 1.43]. The RF pattern was associated with higher probability of overweight/ obesity [1.20, 95\% CI: 1.08, 1.32], high LDL-cholesterol [1.20, 95\% CI:1.07, 1.37], and less likelihood of diabetes [0.87, 95\% CI: 0.77, 0.98]. The FVS pattern was associated with lower probability of overweight/obesity [0.85, 95\% CI: 0.77, 0.92], diabetes [0.88, 95\% CI: 0.80, 0.97], high triglycerides [0.90, 95\% CI: $0.81,1.00]$, and hypertension [0.88, 95\% CI: 0.81, 0.96].

\section{Discussion}

This study evaluated the relationship between dietary quality and food patterns of Filipino adults and the rising prevalence of selected cardiometabolic NCD risk factors. Dietary quality was derived from the national food consumption survey adopting the AHEI-2010 pattern as standard. The respondents in this study reported poor 


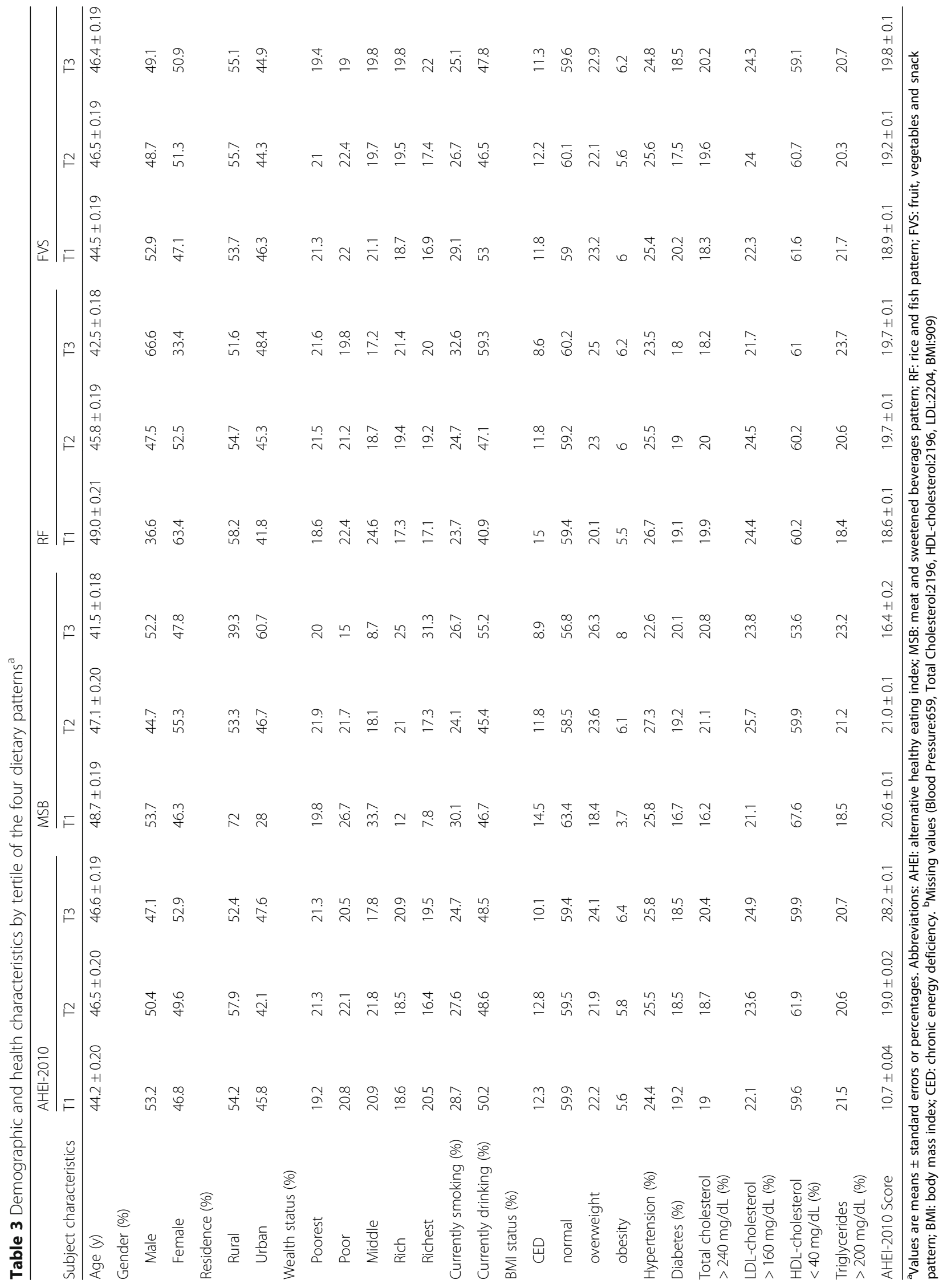




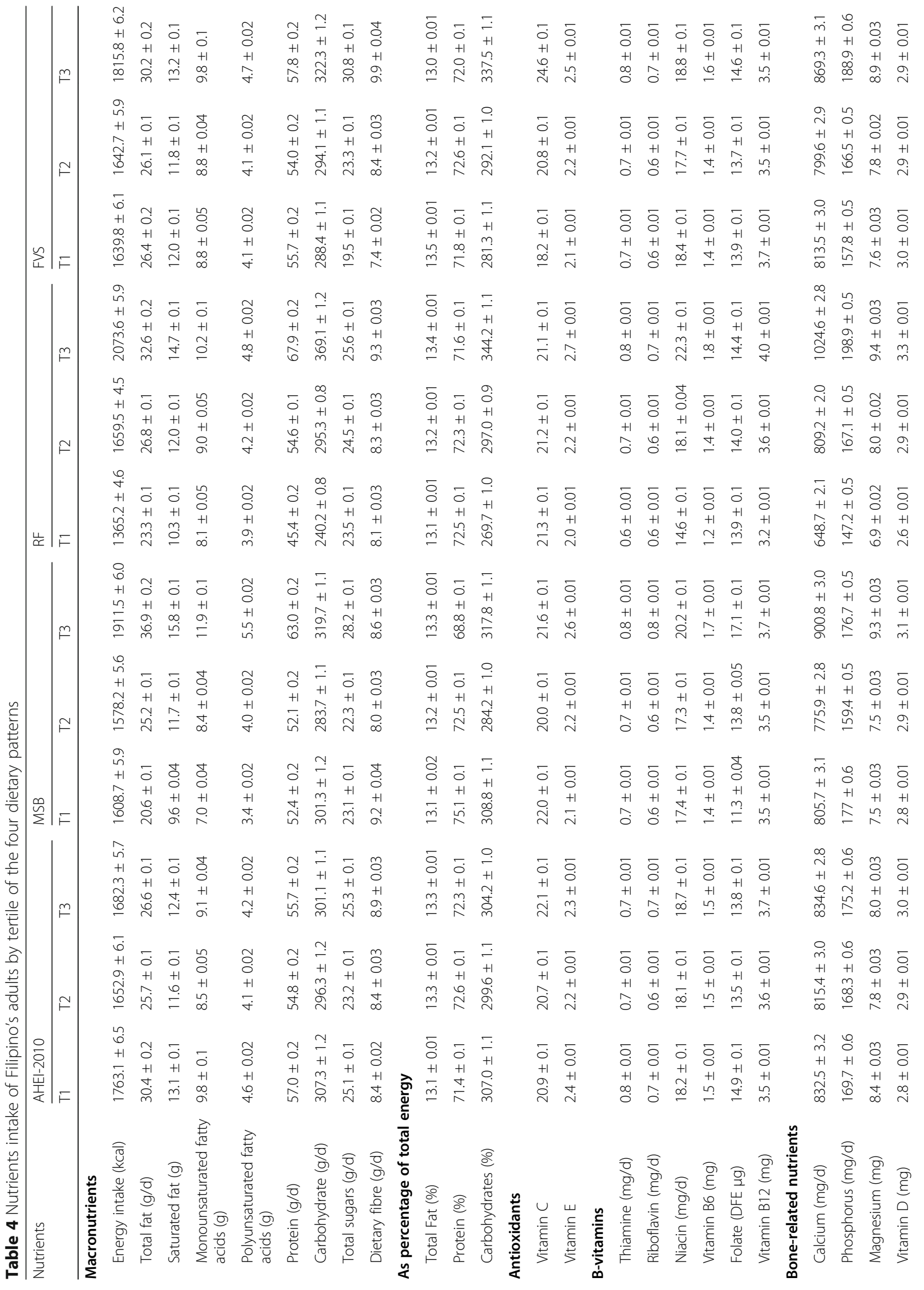




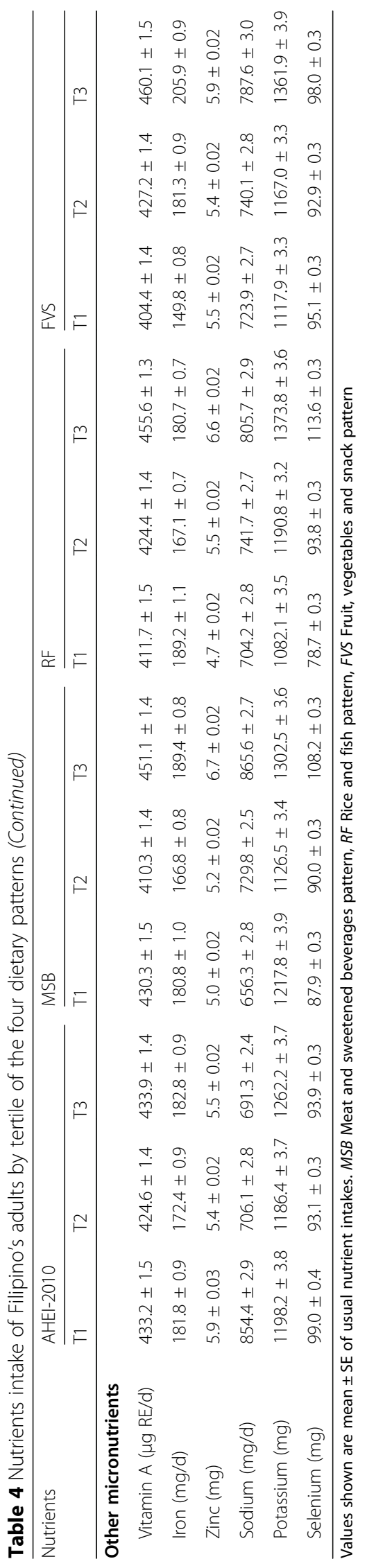




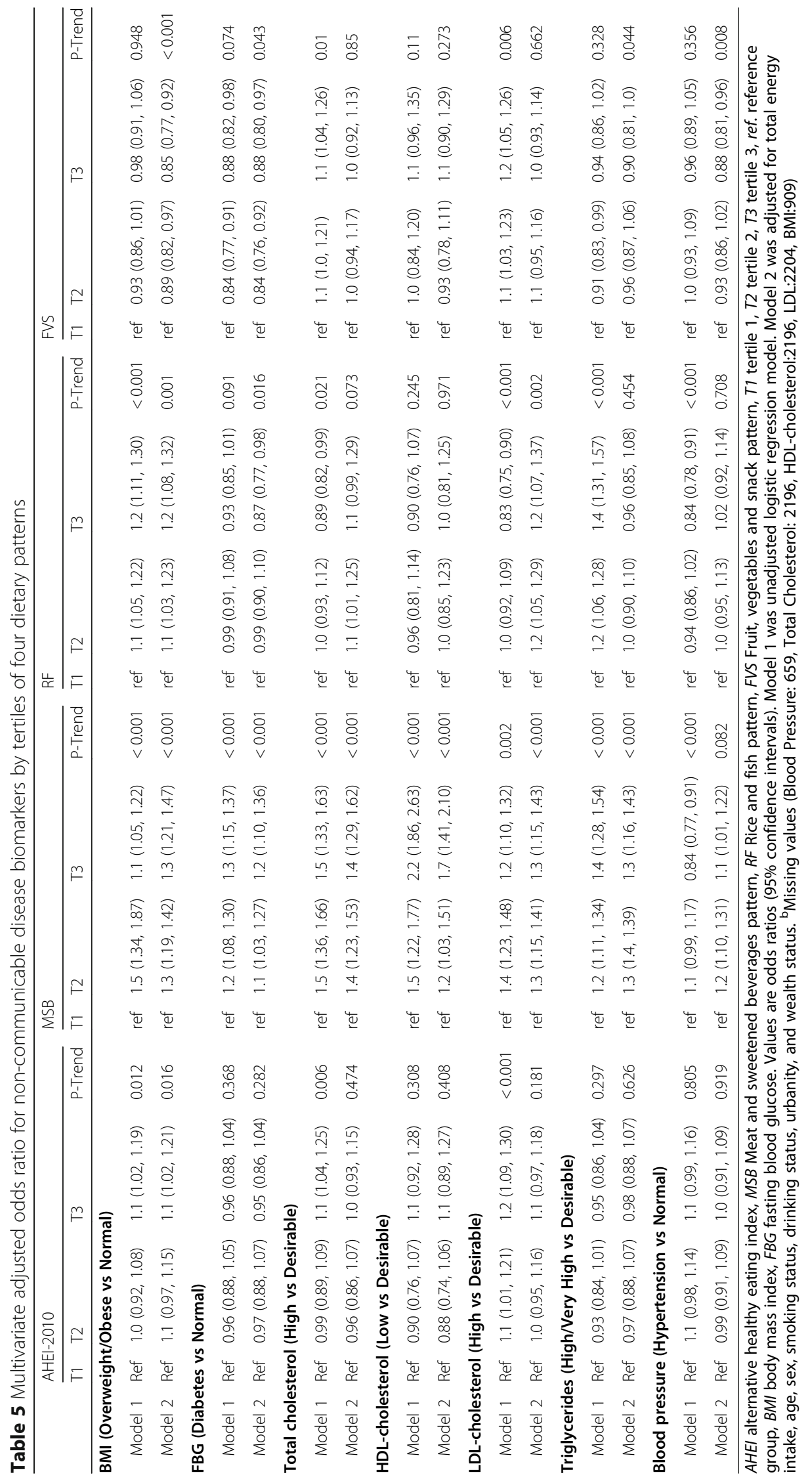


overall diet quality as illustrated by the very low mean score of AHEI-2010 of less than 20 out of 100. This is very low compared with the findings in many other countries: Brazilian population had a mean adapted HEI-2015 of 45.7; among Americans, the mean AHEI2010 was 52.4 for men and 47.6 for women out of 110 ; the Chinese had a mean AHEI-2010 of 42.2 for men and 43.8 for women out of 80; and the finding among Singaporeans revealed that the median quintile range of AHEI-2010 was 48.1-51.6 out of 110 total score [12, 17-19]. Very low consumption of vegetables, fruits, and whole grains were the main contributing factors for the poor quality of diet, and these could be due to several reasons: high price, poor availability, low accessibility and possible contamination of pesticides, lack of knowledge on the benefits of these foods, and no time to cook especially among working adults [20, 21]. In a previous study, better diet quality is seen in women compared with men due to higher awareness and better nutrition knowledge of women than men and several studies also point out that women seek nutrition counselling more frequently than men do [22]. In this present study only a slight difference in AHEI is seen among women (19.7) and men (18.9). This insignificant difference can be attributed to the varied modes of acquiring information about nutritious diet on different social media platforms.

Due to lack of variability in the studied sample using the hypothesis-based approach, AHEI-2010 score was not associated with many socio-demographic characteristics and the selected CMS. Therefore, we explored dietary patterns which could be potentially more meaningful to the local diet with a data-driven approach, PCA. Three major dietary patterns were identified, a meat and sweetened beverages pattern (MSB), a rice and fish pattern (RF), and a fruits, vegetables and snack pattern (FVS).

Our respondents who consume a MSB and RF patterns (highest tertile) are more likely to be younger, urban residents, and from the rich and richest wealth quintile. This is in conformance with an earlier study which revealed that dietary patterns differ between urban and rural areas due to differences in educational attainment, financial resources, and access to healthier foods [23, 24]. Furthermore, urban areas have higher accessibility to a wide range of processed and traditional high-sugar, high-fat snack foods and beverages [25]. The Food and Agriculture Organization statistics also showed that fish consumption in urban areas stood at $14.5 \mathrm{~kg}$ per capita per year compared to $11 \mathrm{~kg}$ per capita per year in rural areas, this is in line with our finding that the RF pattern are more likely to be consumed by urban residents. Also in our study, respondents who are in the highest tertile of the FVS patterns are more likely to be from the richest wealth quintile. This is in agreement with the study in Korea where fruit consumption is associated with higher income and educational level [26]. The same findings were seen in Australiaand China $[27,28]$.

In terms of association with cardiometabolic NCD risk factors, the MSB pattern were associated with a higher risk of various metabolic disorders including overweight and obesity, diabetes, and dyslipidemia, possibly through higher intakes of energy, fat, sugar and sodium. The RF diet also showed an association with cardiometabolic risks. It has been found that fish and rice are contaminated with methylmercury $(\mathrm{MeHg})$ when produced in polluted areas. The chemical form of $\mathrm{MeHg}$ in fish tissue has recently been identified as attached to the thiol group of the cysteine residues in fish protein [29], which are not removed and destroyed by any cooking or cleaning processes. Similarly rice cultivated in $\mathrm{Hg}$ contaminated areas can contain relatively high levels of $\mathrm{MeHg}$ [30-34] and the main route of human $\mathrm{MeHg}$ exposure is related to frequent rice consumption [32]. A body of evidence was developed that addresses potential associations between $\mathrm{MeHg}$ and a range of cardiovascular effects. These include cardiovascular disease (coronary heart disease, acute myocardial infarction (AMI), ischemic heart disease), blood pressure and hypertension effects, and alterations in heart rate variability $[35,36]$. There are strong evidences for causal associations with cardiovascular disease, particularly AMI in adult men [37-40]. On the contrary, the FVS pattern was associated with lower risk of overweight, obesity, diabetes, dyslipidemia, and hypertension, which could be mediated through higher intakes of various beneficial nutrients including fiber, folate, calcium, potassium and magnesium.

A high consumption of sugar-sweetened beverages is evident in this study. Increased consumption of free sugars is particularly indicated in the form of sugarsweetened beverages. Sugar-sweetened beverages usually contain added sugar such as sucrose or high fructose corn syrup. Every $330 \mathrm{ml}$ or $12 \mathrm{oz}$. portion of sugarsweetened carbonated soft drinks typically contains $35 \mathrm{~g}$ (around nine teaspoon) of sugars and provide approximately $140 \mathrm{kcal}$ of energy, but generally with little value of other nutrients [41]. As part of an unhealthy dietary pattern, this may have an effect on increased blood sugar, LDL-cholesterol and triglycerides. Thus, poor diet contributes to the occurrence of a cluster of disorders known as the metabolic syndrome: abdominal obesity, hypertension, dyslipidemia, and disturbed metabolism of glucose or insulin [42]. The presence of the metabolic syndrome increases the risk of developing NCDs such as cardiovascular diseases, diabetes, chronic respiratory diseases, and cancer $[43,44]$.

The prevalence of cardiometabolic NCD risk factors continues to rise in the Philippines and this is 
compounded by the practice of unhealthy lifestyle behaviours. In 2013, the prevalence of high fasting blood glucose among adults was 5.6\%, and this has increased to $7.9 \%$ in 2018 [45, 46].

Additionally, the prevalence remained high for elevated blood pressure (19.2\%) (NNS 2018 data), total cholesterol (18.6\%), LDL-cholesterol (21.9\%) and triglycerides (17.7\%). (NNS 2013 data) The key dietary components that lower cholesterol and triglycerides include increased consumption of fruits, vegetables, and whole grains instead of highly refined ones and plant-based protein $[47,48]$. However, these are consumed in very small amounts in the studied population. Fruit and vegetable consumption of Filipino adults was only at $41 \mathrm{~g}$ and $114 \mathrm{~g}$ per capita respectively; further, only about $9.9 \%$ of the population were consuming whole grains.. In our study, the respondents who consumed a FVS pattern was observed to have an overall lower metabolic risk profile, which further corroborates the importance of promoting higher consumption of fruits, vegetables, and healthy snacks among the Filipino adults. Besides unhealthy diet, the prevalence of current smokers during the study period was $25.4 \%$; binge drinkers was $56.2 \%$; and physical inactivity was $45.5 \%$, and these numbers remained high in the latest national survey conducted in 2018. Promoting healthy lifestyle is indeed very much needed.

To our knowledge, our study is the first one to use recent nationally representative data to characterize the dietary patterns of adults in the Philippines. The utilization of both a priori defined index (AHEI-2010) and posteriori derived dietary patterns (PCA) provided complementary and comprehensive assessment of the Filipino dietary quality and food consumption patterns. However, this study has several limitations. Firstly, the dietary data collection using $24-\mathrm{h}$ recalls is subject to measurement errors from the subjects' recall and estimation of consumption portions. Secondly, the lack of trans-fat information in our food composition database limits our ability to assess trans-fat as a component of AHEI-2010 in association with cardiometabolic risk factors. Lastly, the cross-sectional design of the survey prohibits us from drawing conclusions about the causal relationship between the observed dietary pattern and the cardiometabolic NCD risk factors. Future prospective studies are warranted to corroborate the findings of the present study.

\section{Conclusions}

This study first characterized the diet of Philippines adults using the AHEI-2010 method, which suggested overall poor quality of diet. Three major dietary patterns in the studied population were then identified using a data-driven approach (PCA). Diet quality of Filipino adults is extremely poor. Meat and sweetened beverages and rice and fish patterns were associated with a higher risk of all the cardiometabolic NCD indices, while a fruits, vegetables and snack pattern was associated to a lower risks of cardiometabolic risks. Identifying healthy and detrimental dietary patterns in the local diet could be informative for future local-based dietary recommendation and area-specific intervention programs.

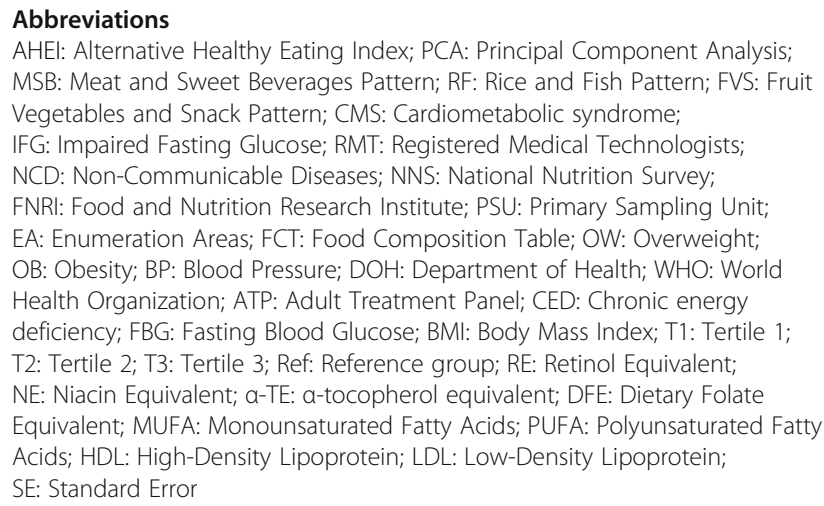

\section{Acknowledgements}

The research described here was a collaborative study of 2 organizations: The Department of Science and Technology, Food and Nutrition Research Institute (DOST- FNRI), Philippines and Nestlé Research Center (Nestec S.A.), Switzerland. The authors would like to acknowledge Mario V. Capanzana, Ph. D, for the support during the preparation of this manuscript.

\section{Authors' contributions}

Imelda Angeles-Agdeppa, Ye Sun, conceptualized and designed the study, interpreted the data, drafted the initial manuscript, and approved the final manuscript as submitted. Keith V. Tanda did the statistical data processing and analysis, and. All authors proof-read and approved the manuscript.

\section{Funding}

The research described here was a collaboration of 2 organizations: The Department of Science and Technology, Food and Nutrition Research Institute (DOST- FNRI), Philippines (data collection and analyses), and Nestlé Research (Nestec S.A.), Switzerland, (funding source and study conceptualization).

\section{Availability of data and materials}

All data generated or analysed during this study are included in this published article and its supplementary information files.

Ethics approval and consent to participate

The Ethics Committee of FNRI approved the survey protocol. All surveyed households provided informed consent prior to participation.

Consent for publication

Not applicable.

\section{Competing interests}

The authors declare no conflict of interest. Y.S. is an employee of Nestec S.A., Switzerland. The opinions expressed in this article are those of the authors alone and do not necessarily reflect the views or recommendations of their affiliations. 
Received: 13 April 2020 Accepted: 27 July 2020 Published online: 03 August 2020

\section{References}

1. Shivastava AK. Challenge in the treatment of Cardiometabolic syndrome. Indian J Phramacol. 2012:44(2):155-6.

2. Mayo clinic. Cardiometabolic program. Overview. http://www.mayoclinic org/departments-centers/cardiometabolic-program/overview/ovc-20442218. Accessed on 1 Sept 19.

3. World Health Organization. Equity, social determination and public health programmers. Geneva: World Health Organization (WHO) Press; 2010.

4. World Health Organization. World Health Organization-Non-Communicable Diseases (NCD) Country Profile. 2018.

5. Department of Health Website. https://www.doh.govph/Statistics/LeadingCauses-of-Mortality. Accessed on 1 Sept 19.

6. Tacio, H.D. (2016). Heart failure getting prevalent among Filipinos. Business Mirror. Available online: https://businessmirror.com.ph/2016/11/30/heartfailure-getting-prevalent-among-filipinos. Accessed on 1 Sept 19.

7. Tumanan-Mendoza BA, Mendoza VL, Bermudez-Delos Santos AA, Punzalan FE, Pestano NS, et al. Epidemiologic burden of hospitalization for congestive heart failure among adults aged $>19$ years in the Philippines. Heart Asia. 2017;1:76-80.

8. Cecchini M, Sassi F, Lauer JA, Lee YY, Guajardo-Barron V, et al. Tackling of unhealthy diets, physical inactivity, and obesity: health effects and costeffectiveness. Lancet. 2010;376:1775-84.

9. Naicker A, Venter CS, Maclntyre UE, Ellis S. Dietary quality and patterns and non-communicable disease risk of an Indian community in KwaZulu-Natal. J Health Popul Nutr. 2015;33:22.

10. Non-communicable disease a growing problem in the Philippine. Oxford Business Group. https://oxfordbusinessgroup.com/analysis/out-shapechanges-lifestyle-have-led-growin-concerns-over-non-communicablediseases. Accessed on 1 Sept 19.

11. FAO/INFOODS. Guidelines for Food Matching. Version 1.2 (2012). Rome: FAO; 2012.

12. Chiuve SE, Fung TT, Rimm EB, Hu FB, McCullough ML, Wang M, Stamfer MJ, Willett WC. Alternative dietary indices both strongly predict risk of chronic disease. J Nutr. 2012;142:1009-18.

13. Physical status: the use and interpretation of anthropometry. Report of a WHO Expert Committee. WHO Technical Report Series No. 854. Geneva: World Health Organization; 1995.

14. James PA, Oparil S, Carter BL, et al. 2014 Evidence-Based Guideline for the Management of High Blood Pressure in Adults: Report From the Panel Members Appointed to the Eighth Joint National Committee (JNC 8). JAMA. 2014;311(5):507-20.

15. World Health Organization. Definition and diagnosis of diabetes mellitus and intermediate hyperglycaemia: report of a WHO/IDF consultation. 2006

16. National Cholesterol Education Program (NCEP) Expert Panel on Detection, Evaluation, and Treatment of High Blood Cholesterol in Adults (Adult Treatment Panel III). Third report of the National Cholesterol Education Program (NCEP) Expert Panel on detection, evaluation, and treatment of high blood cholesterol in adults. Circulation. 2002;106:3143.

17. De Paula Matos Souza J, Magela de Lima M, Martins Horta P. Diet Quality among the Brazilian Population and Associated Socioeconomic and Demographic Factors: Analysis from the National Dietary Survey 2008-2009. J Acad Nutr Diet. 2019;119(11):1866-74.

18. Yu D, Zhang X, Xiang YB, Yang G, Li H, Gao YT, et al. Adherence to dietary guidelines and mortality: a report from prospective cohort studies of 134,000 Chinese adults in urban Shanghai. Am J Clin Nutr. 2014;100:693-700

19. Neelakantan N, Koh WP, Yuan JM, Dam RM. Diet-quality indexes are associated with a Loweer risk of cardiovascular, respiratory, and all-cause mortality among Chinese adults. J Nutr. 2018;148:1323-32.

20. Filipino need more Fruits and Vegetables. http://manilastandard.net/ lifestyle/wellness-environment/211700/filipinos-need-more-fruits-andvegetables.html. Accessed on 1 Sept 19.

21. Brownlee IA, Durukan E, Masset G, Hopkins S, Tee ES. An overview of whole grain regulations, Recommendations and Research across Southeast Asia. Nutrients. 2018;6:752

22. Kiefer I, Rathmanner T, Kunze M. Eating and dieting differences in men and women. J Mens Health Gender. 2005;2(2):194-201.
23. Savoca MR, Arcury TA, Leng X, Bell RA, Chen H, Anderson A, et al. The diet quality of rural older adults in the south as measured by healthy eating Index-2005 varies by ethnicity. J Am Diet Assoc. 2009;109(12):2063-7.

24. Miller PE, Morey MC, Hartman TJ, Snyder DC, Sloane R, Cohen HJ, DemarkWahnefried W. Dietary Patterns Differ between Urban and Rural Older, Long-Term Survivors of Breast, Prostate, and Colorectal Cancer and Are Associated with Body Mass Index. J Acad Nutr Diet. 2012;112(6):824-31.

25. Nurwanti E, Hadi H, Chang JS, Chao JCJ, Paramashanti BA, Gittelsohn J, Bai $\mathrm{CH}$. Rural-urban differences in dietary behavior and obesity: results of the Riskesdas study in 10-18-year-old Indonesian children and adolescents. Nutrients. 2019;11(11):2813.

26. Choi A, Ha K, Joung $H$, Song Y. Frequency of consumption of whole fruit, not fruit juice, is associated with reduced prevalence of obesity in Korean adults. J Acad Nutr Diet. 2019:199(11):1-10.

27. Giskes K, Turrel G, Patterson C, Newman B. Socio-economic differences in fruit and vegetable consumption among Australian adoloscents and adults. PublicHealth Nutr. 2002;5(5):663-9.

28. Li YC, Jiang B, Zhang M, Huang ZJ, Deng Q, Zhou MG, et al. Vegetable and fruit consumption among Chinese adults and Associtaed factors: a nationally representative study of 170,847 adults. Biomed Environ Sci. 2017; 30(12):140-7.

29. Harris HH, Pickering IJ, George GN. The chemical form of mercury in fish. Science. 2003:301:1203.

30. Horvat M, Nolde N, Fajon V, et al. Total mercury, methylmercury and selenium in mercury polluted areas in the province Guizhou, China. Sci Total Environ. 2003:304:231-56.

31. Qiu G, Feng X, Wang S, Shang L. Environmental contamination of mercury from hg-mining areas in Wuchuan, northeastern Guizhou, China. Environ Pollut. 2006;142:549-58.

32. Feng X, Li P, Qiu GL, et al. Human exposure to methylmercury through rice intake in mercury mining areas, Guizhou province, China. Environ Sci Technol. 2008;42:326-32.

33. Li P, Feng XB, Qiu GL, et al. Mercury exposure in the population from Wuchuan mercury mining area, Guizhou, China. Sci. Total Environ. 2008;395:72-9.

34. Qiu G, Feng X, Li P. Methylmercury accumulation in rice (Oryza sativa L) grown at abandoned mercury mines in Guizhou, China. J Agr Food Chem. 2008;56:2465-8.

35. Chan HM, Egeland GM. Fish consumption, mercury exposure, and heart diseases. Nutr Rev. 2004;62:68-72.

36. Stern AH. A review of the studies of the cardiovascular health effects of methylmercury with consideration of their suitability for risk assessment. Environ Res. 2005;98:133-42.

37. Guallar E, Sanz-Gallardo MI, Van't Veer $P$, et al. Mercury fish oils, and the risk of myocardial infarction. N Engl J Med. 2002;347:1747-54.

38. Rissanen T, Voutilainen S, Nyyssonen $\mathrm{K}$, et al. Fish oil-derived fatty acids, docosahexaenoic acid and docosapentaenoic acid, and the risk of acute coronary events: the Kuopio ischaemic heart disease risk factor study. Circulation. 2000;102:2677-9.

39. Salonen JT, Seppanen K, Lakka TA, et al. Mercury accumulation and accelerated progression of carotid atherosclerosis: a population-based prospective 4-year follow-up study in men in eastern Finland. Atherosclerosis. 2000;148:265-73.

40. Virtanen JK, Voutilainen S, Rissanen TH, et al. Mercury fish oils, and risk of acute coronary events and cardiovascular disease, coronary heart disease, and all-cause mortality in men in eastern Finland. Arterioscl Throm Vas. 2005;25:228-33.

41. World Health Organization (WHO). Reducing consumption of sugar-sweetened beverages to reduce the risk of unhealthy weight gain in adults. https://www. who.int/elena/bbc/ssbs_adult_eight/en/. Accessed on 1 Sept 19.

42. Olatona FA, Onabanjo OO, Ugbaja RN, Nnoaham KE, Adeleken DA. Dietary habits and metabolic risk factors for non-communicable diseases in a university undergraduate population. J Health Populat. 2018;37:21.

43. Grundy SM, Cleeman II, Daniels SR, Donato KA, Eckel RH, Franklin BA, et al. Am Heart Assoc. 2005;17:2735-52.

44. Agyei-Mensah S, de-Graft Aikins A. Epidemiological transition and the double burden of disease in Accra, Ghana. J Urban Health. 2010;5:879-97.

45. Philippine Nutrition Facts and Figures 2013: 8th National Nutrition Survey Overview. http://enutrition.fnri.dost.gov.ph/assets/uploads/publications/ Overview_8thNNS_050416.pdf. Accessed on 1 Sept 19.

46. Philippine Nutrition Facts and Figures 2018: Expanded National Nutrition Survey Health and Nutritional Status of Filipino Adults and 
Elderly. https://www.fnri.dost.gov.ph/images//sources/eNNS2018/Adults_ and_Elderly.pdf. Accessed on 1 Sept 19.

47. 11 Foods that lower cholesterol. https://www.health.harvard.edu. Accessed on 1 Sept 19 .

48. Viuda-Martos M, López-Marcos MC, Fernández-López J, Sendra E, LópezVargas JH, Pérez-Álvarez JA. Role of fiber in cardiovascular diseases: a review. Compr Rev Food Sci Food Saf. 2010;9:240-58.

\section{Publisher's Note}

Springer Nature remains neutral with regard to jurisdictional claims in published maps and institutional affiliations.

Ready to submit your research? Choose BMC and benefit from:

- fast, convenient online submission

- thorough peer review by experienced researchers in your field

- rapid publication on acceptance

- support for research data, including large and complex data types

- gold Open Access which fosters wider collaboration and increased citations

- maximum visibility for your research: over $100 \mathrm{M}$ website views per year

At BMC, research is always in progress. 